\title{
Finally, after decades, immune checkpoint inhibitors dethroned the standard of care of small-cell lung cancer
}

\author{
Khalil Saleh ${ }^{1}$, Nadine Khalife-Saleh ${ }^{1}$ \& Hampig Raphael Kourie ${ }^{*, 1}$ \\ ${ }^{1}$ Hematology-Oncology Department, Faculty of Medicine, Saint Joseph University of Beirut, Lebanon \\ *Author for correspondence: hampig.kourie@hotmail.com
}

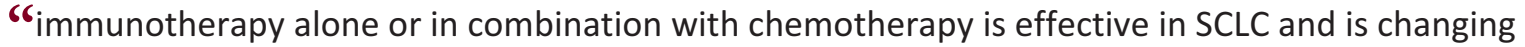
the treatment sequencing in extensive-stage disease. Efforts are needed to define the adequate strategy of management of SCLC"

First draft submitted: 13 January 2019; Accepted for publication: 31 January 2019; Published online: 12 March 2019

Keywords: extensive-stage SCLC • immune checkpoint inhibitor • PD-1 inhibitor • PD-L1 inhibitor • tumor mutational burden

Small-cell lung cancer (SCLC) is an aggressive disease associated with poor prognosis. It represents around 15\% of all lung cancers [1]. $60 \%$ of patients have extensive-stage disease at diagnosis. The majority of patients (95\%) diagnosed with SCLC are current or former smokers [2]. Platinum chemotherapy (cisplatin or carboplatin) with etoposide remains the standard of care first-line treatment in patients with extensive-stage SCLC with a median overall survival (OS) of approximately 10 months [3]. Despite initial overall response rate (ORR) of $67 \%$ to this first-line treatment, limited progress has been made in more than 20 years. Topotecan is the only US FDA- and EMA-approved second-line chemotherapy in the treatment of extensive-stage SCLC after failure of platinum-based chemotherapy [4]. Immune checkpoint inhibitors (ICIs) have been approved by the US FDA in the treatment of several solid tumors such as non-small-cell lung carcinomas (NSCLC), clear cell renal carcinomas, metastatic melanoma, head and neck squamous cell carcinoma (HNSCC) and other subtypes of carcinoma [5]. ICIs have been also evaluated in patients with SCLC and will be probably approved by the FDA in the first-line setting in combination with chemotherapy. We will highlight here the new data of ICI in first-line and second-line setting in SCLC, the available predictive biomarkers and the future perspectives of the management of this disease.

\section{Immune checkpoint inhibitors in first-line setting}

Recently, Horn et al. reported the results of the IMpower133 study, a multinational, double-blind, randomized, placebo-controlled Phase III trial. This study compared atezolizumab, a programmed-death ligand 1 (PD-L1) inhibitor, plus carboplatin and etoposide to placebo plus carboplatin and etoposide followed by maintenance with either atezolizumab or placebo in patients with extensive-SCLC who have not been treated for advanced disease. This is the first published Phase III trial which evaluated the combination of ICI and chemotherapy in patients with advanced SCLC and met its primary end point in terms of overall survival (OS) and progression-free survival (PFS) in first-line setting. Median OS was 12.3 months in the atezolizumab group versus 10.3 months in the placebo group (hazard ratio [HR], 0.70; 95\% confidence interval [CI], 0.54-0.91; $\mathrm{p}=0.007$ ). Median PFS was 5.2 months in the experimental arm compared with 4.3 months in the control arm (HR: 0.77; 95\% CI: 0.62-0.96; $\mathrm{p}=0.02)[6]$.

The results of IMpower133 were concordant with the previously published data concerning the combination of immunotherapy with chemotherapy in previously untreated advanced nonsquamous NSCLC and HNSCC. In the KEYNOTE-189 Phase III trial, the addition of pembrolizumab to pemetrexed and platinum-based chemotherapy was significantly superior to chemotherapy alone in patients with previously untreated metastatic nonsquamous NSCLC without EGFR and ALK mutation in terms of PFS and OS. The median PFS was 8.8 months in the pembrolizumab group compared with 4.9 months in the chemotherapy group (HR: 0.52; 95\% CI: 0.43-0.64; 
$\mathrm{p}<0.001$ ). The 12 -month OS rate was $69.2 \%$ in the experimental arm versus $49.4 \%$ in the control arm (HR: 0.49; 95\% CI: 0.38-0.64; p < 0.001) [7]. Similarly, Socinski et al. reported, in the IMpower150 study, that the addition of atezolizumab to bevacizumab and chemotherapy (paclitaxel plus carboplatin) significantly prolonged the median PFS and OS compared with bevacizumab and chemotherapy in patients with previously untreated metastatic nonsquamous NSCLC. The median PFS was 8.3 months in the atezolizumab group versus 6.8 months in the control group (HR: 0.62; 95\% CI: 0.52-0.74; $\mathrm{p}<0.001$ ) and the medias OS was 19.2 months in the experimental arm versus 14.7 months in the control arm (HR: 0.78; 95\% CI: 0.64-0.96; $p=0.02$ ) [8]. Furthermore, at last ESMO 2018, Burtness et al. reported the results of the KEYNOTE-048 Phase III trial in patients with previously untreated metastatic HNSCC. Pembrolizumab plus chemotherapy (5-FU and cisplatin or carboplatin) was compared with the standard of care regimen (5-FU, cisplatin or carboplatin and cetuximab). The experimental arm was associated with significantly prolonged OS compared with the standard arm (13.0 vs 10.7 months, respectively; $\mathrm{p}=0.0034$ [ [9].

In a Phase II trial evaluating pembrolizumab, a programmed-death 1 (PD-1) inhibitor, as maintenance therapy after induction therapy with etoposide and platinum in patients with extensive-stage SCLC, the median PFS from the beginning of maintenance was 1.9 months and the median OS was 9.6 months [10]. This suggests that ICI in combination with chemotherapy is efficacious in the treatment of extended stage SCLC. An ongoing randomized, double-blind, placebo-controlled Phase III trial is evaluating the addition of pembrolizumab to etoposide and platinum followed by maintenance pembrolizumab in patients with extensive-stage SCLC in the KEYNOTE-604 (NCT03066778).

Ipilimumab, a cytotoxic T-lymphocyte-associated antigen 4 (CTLA-4) inhibitor, was the first ICI evaluated as first-line treatment in combination with etoposide and platinum in patients with extensive-stage SCLC. The randomized, double-blind, placebo-controlled, Phase III trial compared the ipilimumab plus etoposide and platinum versus placebo plus etoposide and platinum with maintenance with either ipilimumab or placebo. The study failed to meet its primary end point which was OS. Median OS was 11.0 months in the ipilimumab group compared with 10.9 months in the placebo group (HR: 0.94; 95\% CI: 0.81-1.09; $\mathrm{p}=0.3775$ ). Median PFS was 4.6 months in the experimental group versus 4.4 months (HR: 0.85; 95\% CI: 0.75-0.97) [11]. These results may suggest that CTLA-4 inhibitor was not effective in the treatment of extensive-stage SCLC.

\section{Immune checkpoint inhibitors in second-line setting or more}

The results of CheckMate-032 study contributed for the first FDA-accelerated approval for ICI (nivolumab, a PD-1 inhibitor, alone or in combination with ipilimumab) in third-line treatment of metastatic SCLC in August 2018 regardless of PD-L1 status. This study evaluated nivolumab alone or in combination with ipilimumab. The ORRs were $12 \%$ in the nivolumab alone group and $22 \%$ in the combination group. Grade 3-4 adverse events were observed in $12 \%$ of patients treated with nivolumab and $37 \%$ of patients treated with nivolumab and ipilimumab [12]. The CheckMate331, an open-label, randomized Phase III ongoing trial is comparing nivolumab with topotecan or amrubicin in patients with relapsed SCLC after failure of first-line platinum chemotherapy with primary end point being the OS (NCT02481830).

Ott $e$ al. presented the preliminary results of the SCLC cohort of KEYNOTE-028 evaluating pembrolizumab in patients with PD-L1-expressing SCLC. The ORR was 33.3\% (8/24, one complete response and seven partial response) [13]. At the last ASCO 2018, Chung et al. reported the results of the SCLC cohort in the Phase II kEYNOTE-158 which evaluated pembrolizumab as monotherapy in patients with advanced incurable SCLC who progressed on or failed standard chemotherapy. The objective response rate (ORR) was $18.7 \%$ overall, $35.7 \%$ in patients with PD-L1-positive tumors and 6\% in patients with PD-L1-negative tumors. Median OS was 9.1 months overall, 14.6 months in patients with PD-L1-positive disease and 7.7 months in PD-L1-negative disease. Median PFS was 2.0 months overall, 2.1 months in PD-L1-positive tumors and 1.9 months in PD-L1-negative tumors [14]. These data suggest that pembrolizumab has shown promising activity in relapsed advanced SCLC after failure of standard treatment, especially in patients with PD-L1-positive tumors. An ongoing randomized, open-label, Phase II trial is evaluating pembrolizumab versus topotecan in patients with SCLC after failure of first-line treatment with etoposide and platinum (NCT02963090).

\section{Tumor mutational burden as biomarker of response}

It has been suggested that tumor mutational burden (TMB) may be an independent predictor of response to immunotherapy in diverse cancers, especially in melanoma and NSCLC $[15,16]$. In a study of 151 patients treated 
with immunotherapy, Goodman et al. showed that the response rate to immunotherapy and median PFS were significantly higher in patients with high TMB compared with patients with low-to-intermediate TMB (58 vs $22 \% ; \mathrm{p}=0.0001$ and 12.8 vs 3.3 months; $\mathrm{p}<0.0001$ respectively) [17]. SCLC is characterized with high TMB due to the nearly universal association of SCLC with smoking [18]. It has been suggested that TMB may be a potential biomarker to predict response to immunotherapy in the treatment of patients with advanced SCLC. An exploratory analysis of the association of TMB and clinical benefit to nivolumab or nivolumab plus ipilimumab has been made. The ORR in nivolumab group was 21.3 versus 6.8 versus $4.8 \%$ in patients with high, medium and low TMB, respectively. The results were most striking in the combination group with an ORR of 46.2 versus 22.2 versus $16 \%$ in the three groups, respectively [19]. However, in the IMpower133 study, Horn et al. reported that TMB was not predictive of response of benefit for the combination of atezolizumab and chemotherapy. The authors concluded that it was due to the high activity and myelosuppression of platinum-based chemotherapy [6].

\section{Conclusion}

In conclusion, immunotherapy alone or in combination with chemotherapy is effective in SCLC and is changing the treatment sequencing in extensive-stage disease. Efforts are needed to define the adequate strategy of management of SCLC and prospective investigations should be done to define predictive factors of response to ICI.

\section{Financial \& competing interests disclosure}

The authors have no relevant affiliations or financial involvement with any organization or entity with a financial interest in or financial conflict with the subject matter or materials discussed in the manuscript. This includes employment, consultancies, honoraria, stock ownership or options, expert testimony, grants or patents received or pending, or royalties.

No writing assistance was utilized in the production of this manuscript.

\section{References}

1. Govindan R, Page N, Morgensztern D et al. Changing epidemiology of small-cell lung cancer in the United States over the last 30 years: analysis of the surveillance, epidemiologic, and end results database. J. Clin. Oncol. 24(28), 4539-4544 (2006).

2. Jackman DM, Johnson BE. Small-cell lung cancer. Lancet Lond. Engl. 366(9494), 1385-1396 (2005).

3. Rossi A, Di Maio M, Chiodini P et al. Carboplatin- or cisplatin-based chemotherapy in first-line treatment of small-cell lung cancer: the COCIS meta-analysis of individual patient data. J. Clin. Oncol. 30(14), 1692-1698 (2012).

4. Farago AF, Keane FK. Current standards for clinical management of small cell lung cancer. Transl. Lung Cancer Res. 7(1), 69-79 (2018).

5. Approvals of FDA-regulated products. www.fda.gov/NewsEvents/ProductsApprovals

6. Horn L, Mansfield AS, Szczęsna A et al. First-line atezolizumab plus chemotherapy in extensive-stage small-cell lung cancer. N. Engl. J. Med. 379(23), 2220-2229 (2018).

7. Gandhi L, Rodríguez-Abreu D, Gadgeel S et al. Pembrolizumab plus chemotherapy in metastatic non-small-cell lung cancer. N. Engl. J. Med. 378(22), 2078-2092 (2018).

8. Socinski MA, Jotte RM, Cappuzzo F et al. Atezolizumab for first-line treatment of metastatic nonsquamous NSCLC. N. Engl. J. Med. 378(24), 2288-2301 (2018).

9. KEYNOTE-048: Phase III study of first-line pembrolizumab (P) for recurrent/metastatic head and neck squamous cell carcinoma (R/M HNSCC) | OncologyPRO. https://oncologypro.esmo.org/Meeting-Resources/ESMO-2018-Congress/KEYNOTE-048-Phase-3-studyof-first-line-pembrolizumab-P-for-recurrent-metastatic-head-and-neck-squamous-cell-carcinoma-R-M-HNSCC

10. Gadgeel SM, Pennell NA, Fidler MJ et al. Phase II study of maintenance pembrolizumab in patients with extensive-stage small cell lung cancer (SCLC). J. Thorac. Oncol. 13(9), 1393-1399 (2018).

11. Reck M, Luft A, Szczesna A et al. Phase III randomized trial of ipilimumab plus etoposide and platinum versus placebo plus etoposide and platinum in extensive-stage small-cell lung cancer. J. Clin. Oncol. 34(31), 3740-3748 (2016).

12. Antonia SJ, López-Martin JA, Bendell J et al. Nivolumab alone and nivolumab plus ipilimumab in recurrent small-cell lung cancer (CheckMate 032): a multicentre, open-label, Phase I/II trial. Lancet Oncol. 17(7), 883-895 (2016).

13. Ott PA, Bang Y-J, Berton-Rigaud D et al. Safety and antitumor activity of pembrolizumab in advanced programmed death ligand 1-positive endometrial cancer: results from the KEYNOTE-028 study. J. Clin. Oncol. 35(22), 2535-2541 (2017).

14. Chung HC, Lopez-Martin JA, Kao SC-H et al. Phase II study of pembrolizumab in advanced small-cell lung cancer (SCLC): KEYNOTE-158. J. Clin. Oncol. 36(15_suppl), 8506-8506 (2018).

15. Snyder A, Makarov V, Merghoub T et al. Genetic basis for clinical response to CTLA-4 blockade in melanoma. N. Engl. J. Med. 371(23), 2189-2199 (2014). 
16. Rizvi NA, Hellmann MD, Snyder A et al. Mutational landscape determines sensitivity to PD-1 blockade in non-small cell lung cancer. Science 348(6230), 124-128 (2015).

17. Steuer CE, Ramalingam SS. Tumor mutation burden: leading immunotherapy to the era of precision medicine? J. Clin. Oncol. 36(7), 631-632 (2018).

18. Alexandrov LB, Nik-Zainal S, Wedge DC et al. Signatures of mutational processes in human cancer. Nature 500(7463), 415-421 (2013).

19. Hellmann MD, Nathanson T, Rizvi $\mathrm{H}$ et al. Genomic features of response to combination immunotherapy in patients with advanced non-small-cell lung cancer. Cancer Cell 33(5), 843-852.e4 (2018). 